\title{
Collaborative Mapping for Pedestrian Navigation in Security Applications
}

\author{
Maria Garcia Puyol, Martin Frassl, and Patrick Robertson \\ German Aerospace Center (DLR), Institute of Communications and Navigation, \\ Muenchnerstrasse 20, 82234 Wessling, Germany, \\ \{maria.garciapuyol, martin.frassl, patrick.robertson\}@dlr.de \\ http://www.kn-s.dlr.de/indoornav/
}

\begin{abstract}
In rescue missions or law enforcement applications, accurate determination of every team member's position and providing this information on a map may significantly improve mutual situation awareness and potentially reduce the risk of accidentally harming a team member. Furthermore, it could help keep track of the areas that have been already visited, helping the coordination of the mission at hand.

Whereas in outdoors environments accurate positioning information can be obtained using a GNSS receivers, in indoor or underground environments GNSS signals are strongly disturbed and other means of localization must be called into play. Foot mounted inertial sensors or IMUs have been one of the proposed solutions, but their performance is prone to errors that grow over time. Only when the map of the environment is provided, can these IMUs perform with high accuracy. But building plans or maps of indoor and underground areas are often unavailable, outdated, incomplete and do not reflect furniture or other obstacles that also constraint the pedestrian's motion. How can a reliable map of an indoor environment be generated?

FootSLAM - Simultaneous Localization and Mapping for pedestrians - is a novel technique based on foot mounted IMUs that measure the pedestrian's steps while walking. These measurements can be used to generate a map of an environment while determining the pedestrian's location within that map. FootSLAM was recently extended to FeetSLAM, the multiuser scenario in which the maps obtained by two or more pedestrians are combined to generate a more extensive and accurate map of the environment.

In this paper we elaborate on different deployment scenarios for FootSLAM and its collaborative counterpart in security and emergency applications, yet to be experimentally validated.
\end{abstract}

Keywords: pedestrian navigation, indoor navigation, SLAM, FootSLAM, collaborative mapping

\section{Introduction: FootSLAM for Pedestrian Navigation}

The problem of pedestrian navigation has received significant attention in the past few years and has been recognized as one of the most fundamental problems 
in the navigation community. Pedestrian navigation is the process by which the location of a pedestrian can be determined over time. This technique is especially important in security and emergency applications, where having precise and realtime knowledge of the location of all the persons involved, i.e. rescue teams and victims, plays a vital role in the success of any emergency operation.

Whereas in outdoor environments the problem can be addressed using satellite navigation receivers (e.g. GPS receivers), their performance is very much impaired in situations suffering from multipath propagation or in indoor and underground environments where the signal strength is not sufficient. This has led to the development of algorithms using other low-cost sensors that the pedestrian wears or carries. Foot mounted MEMS-based inertial sensors (IMUs) have been one of the proposed approaches but their performance suffers from unbounded error growth [1]. Nevertheless, when the environment constrains the movements of the pedestrian and these constraints (i.e. a map) are known, it has been shown that the error can be limited [2], thus allowing stable positioning in two and three dimensions even in the absence of other signals [3], [4].

Accurate positioning information using IMUs can only be obtained when the map of the environment is well-known. However, building plans or maps of indoor or underground areas are often unavailable, private, outdated, incomplete or inaccurate. Moreover, available indoor maps rarely reflect non-wall obstacles that also limit or channel pedestrian motion significantly. If we could rely on a technology that enabled pedestrians to generate a map of an environment while simultaneously providing his position within this map, many location based security applications could be developed.

To this end, FootSLAM - Simultaneous Localization and Mapping for pedestrians - was recently presented [2]. FootSLAM draws on the use of foot-mounted IMUs and builds on SLAM [5] for indoor and urban environments, whereby robots can generate a map of the environment and simultaneously locate themselves within that map. These robots are usually equipped with visual sensors such as laser scanners and cameras, whereas FootSLAM uses only the odometry - the noisy IMU-based measurements of a person's step vectors. Using a RaoBlackwellized Particle Filter, FootSLAM searches over many different odometry error hypotheses finding one which best fits the previous pose history. Hypotheses in which the pedestrian revisits certain areas in the environment are rewarded and over time a reliable 2D map of essentially "walkable areas" is constructed. To help the convergence of the algorithm, FootSLAM allows the inclusion in its estimation process of other data sets obtained while walking in the same building. These data sets are used in the form of a prior map.

Real data from people walking within office environments at five locations have been used to validate the map building and relative localization abilities of FootSLAM [2], [10]. All calculations can be done in real-time. Figures 1 and 2 represent two of the scenarios. Figure 1 shows the FootSLAM map of a building in Vienna that presents a rather interesting layout. Figure 2 depicts two walks within an office building and the original building plan. On the image above, an extensive walk is depicted and the map encompasses almost all offices and other 
walkable areas. Below, the corresponding map of a shorter and less extensive walk is shown.

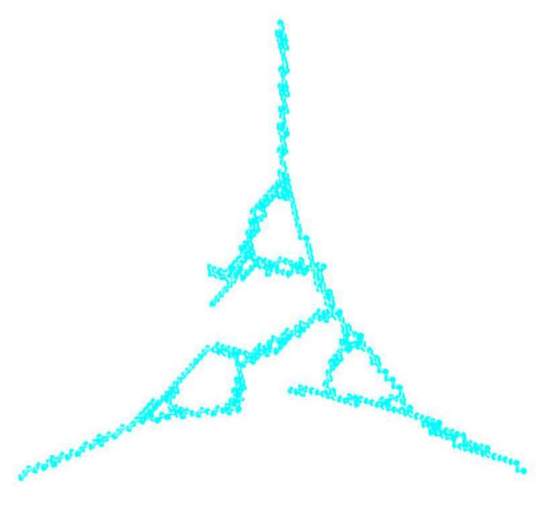

Fig. 1. FootSLAM map obtained from a walk in a building with an unusual layout.

The approach can use other positioning techniques to anchor the map to a global coordinate frame. This can be a GNSS receiver, e.g. GPS, before and after entering a building, or WiFi stations with known positions (see also section 2.3 about sensor fusion), anchoring the map with reasonable position accuracy. Nevertheless, the use of such anchors is not a prerequisite for a valid map to emerge. When no anchor is used, the map has no global reference.

In this contribution we focus on FeetSLAM, the extension of FootSLAM to multiuser cases in which a group of pedestrians collaborate to generate a map of the environment while localizing themselves within that map. This approach is suitable to solve the problem that emergency, security and law enforcement operations often need to face, that is, the problem of having a team of pedestrians moving in an unknown environment and not knowing each other's relative positions.

The rest of this paper is organized as follows: Section 2 starts presenting FeetSLAM as a solution for the problem of pedestrian collaborative mapping. Section 2.1 addresses the different applications for FeetSLAM in online and offline scenarios. Section 2.2 focuses on the possibilities to implement FeetSLAM using either a centralized or a decentralized approach. In Section 2.3, some sensor fusion solutions are proposed to address the generation of a combined map by the collaborating pedestrians and Section 2.4 illustrates how humans and robots can collaborate in the mapping task. Section 2.5 points out the main factors that affect the generation of an accurate combined map. Finally in Section 3, the main conclusions and outlook are presented. 


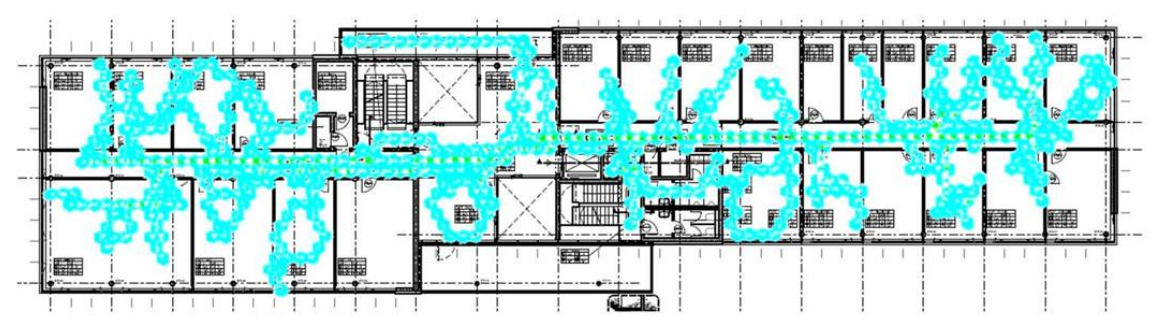

(a) Long walk

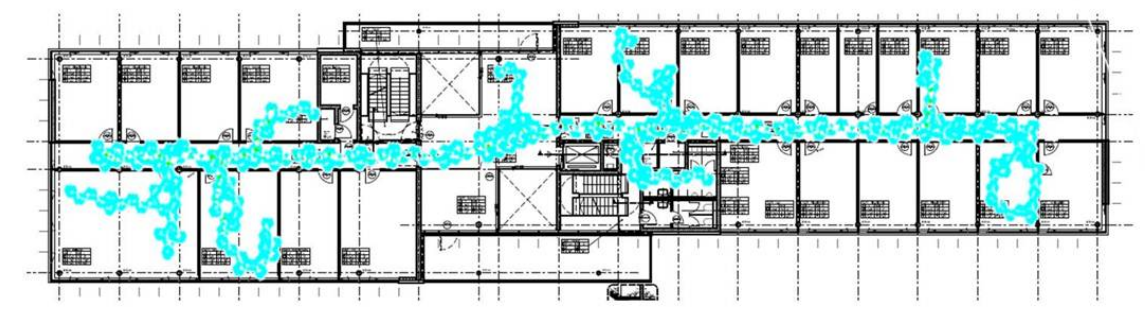

(b) Short walk

Fig. 2. Two examples of FootSLAM maps and building plan overlay for an office building. 2(a) shows the map obtained from a longer and more extensive walk than 2(b).

\section{Collaborative Mapping of Indoor Areas}

The state-of-the-art solutions for collaborative mapping of indoor areas draw on mobile robots that use on-board cameras and laser scanners to detect features in the environment and other robots to compute their relative position and pose [6], [7], [8]. There are also some hybrid solutions where humans and robots collaborate in the mapping task like [9], where the map is generated with the help of RFID tags. To our knowledge, the problem of real-time collaborative mapping by pedestrians using only inertial sensors has not been addressed yet.

FeetSLAM is a new technique [10] that addresses this problem. Following the concept of crowdsourcing [11], [12], in which sourcing tasks traditionally performed by specific individuals are delegated to a group of people or community, FeetSLAM relies on a group of pedestrians to undertake the mapping task. These pedestrians roam through accessible rooms and areas of different levels of a building collecting data by means of an IMU located on their foot or other forms of step measurement with different sensor arrangements. The data is processed by FootSLAM to obtain the individual maps and used in a combined fashion to generate a combined map. In order to do that, the FeetSLAM algorithm needs to find the transformations that place all the considered individual 
maps into the same coordinate system. Once the maps are located within the same coordinate system, their contributions are added to compose the combined map. This combined map can, in turn, be used as a prior map by another data set in another FootSLAM process, provided that data set and prior map are also placed within the same coordinate system. Note that in order to use a map as a prior for a given data set, the map must not contain any contributions from that data set. This is an important requirement that must be held in order to provide the data set with information from other walks while avoiding biasing it with its own contribution.

Collaborative mapping may be processed online in real-time or as an offline mapping process. FeetSLAM was recently implemented and tested in two different office environments in an offline fashion [10], that is, the data are first collected and then processed. Nevertheless, we expect the technique to be sped up to real-time capabilities over time, allowing online location-based services to develop. The next section introduces the main applications for online and offline FeetSLAM.

\subsection{FeetSLAM Online and Offline Applications}

An interesting online use case is the mapping of a building by multiple collaborating pedestrians with the objective of providing immediate map and position information to the other pedestrians or to external users at the time they walk through the building. Feasible scenarios for this use case in the security context are situations like the following:

- Rescue team coordination: A rescue team enters a building through the same or different entrances. Keeping track of the positions helps obtain a status about already visited areas and helps to find areas which have not been explored yet.

- Firefighters safety: Fire fighters operating under respiratory protection in a smoke-filled building are under extreme stress and high risk of hazardous situations. Knowing their exact locations and the shortest route to get there increases the safety during the operations. Figure 3 illustrates such a situation fire fighters often need to face.

- Law enforcement operations: Precise determination of the position of all agents involved in the mission and the knowledge of this position on a map can improve mutual situation awareness and potentially reduce the risk of accidentally harming a team member as well as help coordinate the team.

- Search for missing people in outdoor areas: In areas like deep forest, tunnels or canyons, the use of GNSS is prevented. FeetSLAM can be used to generate a map of the areas as they are visited by the search team and help speed up the search process, specially under bad weather conditions.

In these applications the real-time requirements are severe and usually no prior map data is available, or the map has significantly changed due to the event itself. Doors can be locked or walls might have been destroyed, opening new routes for movement. 


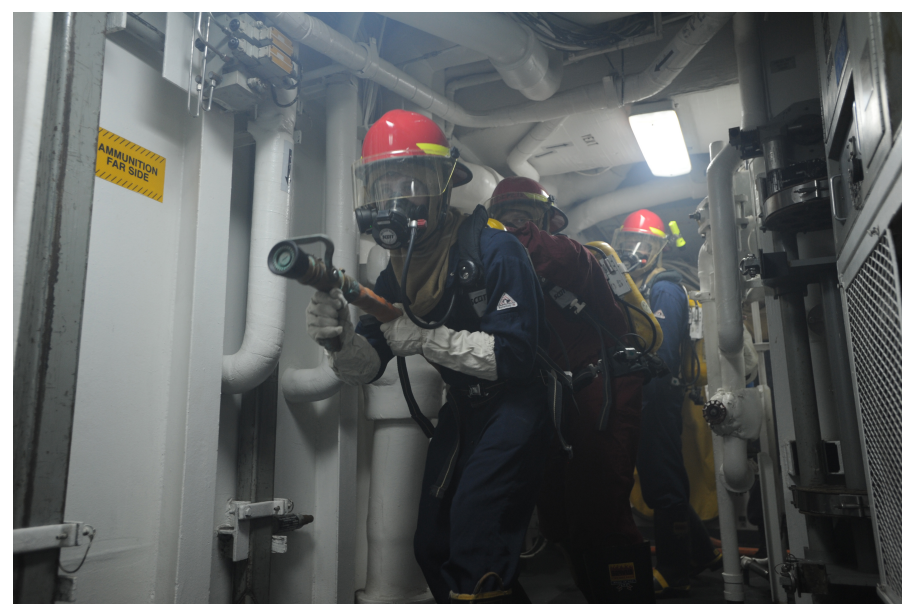

Fig. 3. A team of firefighters undertakes a rescue operation under respiratory protection. The generation of a map of the environment they move in and knowing the position of the rest of the team members can be crucial for the success of the operation. Source: US Navy (Licence CC BY 2.0).

In offline applications we wish to derive a map that can be stored on a server or distributed to localization devices that use it to perform map-aided pedestrian dead reckoning. As more data is collected, the new walks can be incorporated and the maps be refined. Some examples of these offline applications are:

- Localization services for visually impaired people: FeetSLAM maps include information about the layout of the walls, the furniture and other obstacles and can be used by people with reading or vision disabilities, possibly through speech translation.

- Support to mobility-impaired people: These individuals might greatly benefit from FootSLAM maps in which the location of ramps and elevators can be indicated. Also, these maps can help find the shortest path to the destination, avoiding long detours that may cause more discomfort to the pedestrian with the walking disability.

- Intelligence services: Intelligence services can use FootSLAM to generate a map of buildings they are interested in. In this kind of applications, not depending on a external preinstalled infrastructure is a valuable characteristic.

- Evacuation routes: Evacuation routes for events with multitudinous attendance can be planed using FeetSLAM. In case of emergency, these routes can be sent to all the mobile terminals, avoiding locked doors and other obstacles. These applications are especially important in public buildings, airports, and any other buildings with high density of people. The maps can help make the crowd move in a coordinate manner and without colliding with each other, following a swarm behavior.

- Rapid response to accidents: A person among a multitudinous group of persons and located in a huge and complex building structure such as a museum, 
a football stadium or a concert hall, can be reached more quickly thanks to the knowledge of the map of the area, provided the person's location within this map is known. If such person had just suffered a critical incident (e.g. a heart attack), the time to locate him becomes crucial. Furthermore, determining the position of the closest defibrillator, which could also be indicated in the map, can increase the probabilities of saving the person [13].

This offline approach has been implemented in a centralized manner. The data collected by each pedestrian are uploaded to a central server where all the walks are processed in a combined fashion. The next section deepens in both centralized and decentralized approaches for FeetSLAM.

\subsection{Centralized and Decentralized Approaches}

Whereas offline FeetSLAM has been implemented using a central server, the online scenario is also suitable for a decentralized approach.

Centralized approach: The pedestrians only exchange information with the server, but not with each other. The pedestrians can be involved in the combined map generation process in more or less degree:

- The pedestrians are responsible for collecting the odometry data. In this case, the pedestrians are only equipped with an IMU.

- The pedestrians can also generate their own FootSLAM map. To this end, the pedestrians must also carry a FootSLAM processor.

- The pedestrians can also generate the combined map using the information that they obtain from the server. In this scenario, the pedestrians need to be equipped with a FeetSLAM processor.

Likewise, the server can take on one of the following possibilities:

- The server takes all individual data sets or FootSLAM maps and is responsible for the generation of the combined map, which can be then distributed to the pedestrian's terminals.

- The server coordinates the information exchange, but the combined map is computed at each one of the pedestrian's terminal.

The more responsibilities the pedestrians have, the more robust the system is against a failure in the central server or in the communication links.

Decentralized approach: The pedestrians exchange data directly with each other and compute their own individual map and their own version of the combined map. It is possible to exchange odometry data, individual FootSLAM maps or even the combined map. A synchronization system needs to be defined and the amount and order of data transmitted (e.g. the whole map obtained by each pedestrian, or only the last computed portion since the last transmission) has to be determined. This is especially important as the pedestrians are constantly 
moving and the time available for data transmission is limited to the time the two pedestrians are within communication range.

The communication can be done using a centralized network or a decentralized ad hoc network. If the FeetSLAM processing is done centrally, a connection to the central server has to be constantly available, either given implicitly through the centralized network, or through a multihop connection using the decentralized network. If both communication and processing is organized centrally, the system can still benefit from the decentralized network. Map information which has most relevance in the direct vicinity of a person can be exchanged with communication partners located in the vicinity. Communication bandwidth which would be needed to communicate with a central server can be saved. Nevertheless, operational teams are expected to have their own communication network which could be used to distribute maps.

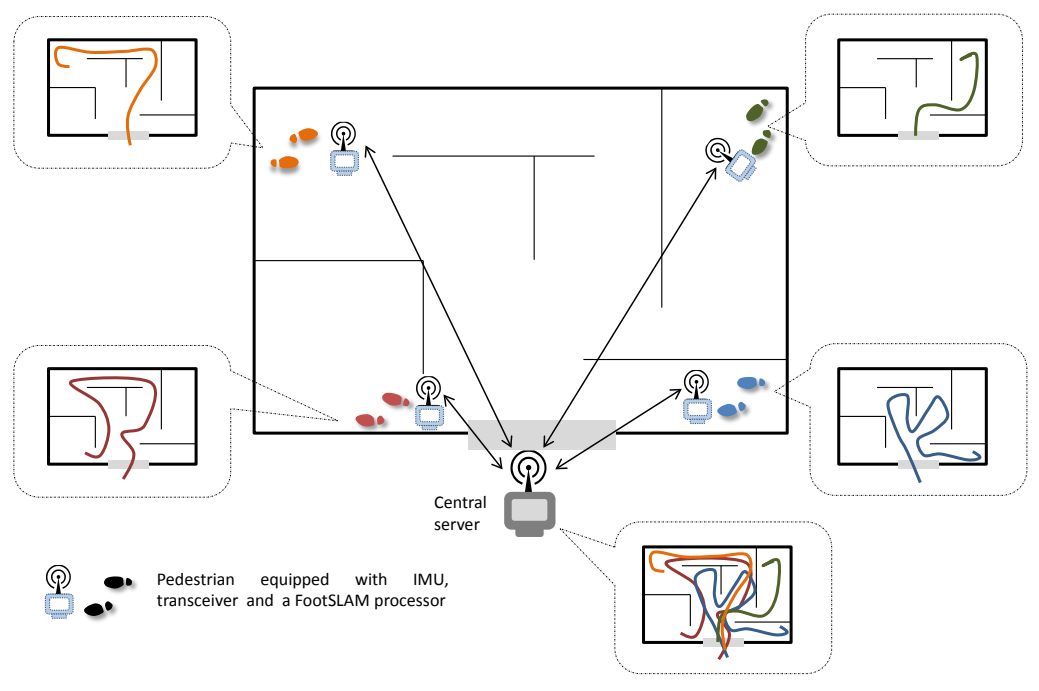

Fig. 4. Centralized FeetSLAM: Four pedestrians have entered the building through the main entrance. Each one of them is equipped with an IMU, a transceiver and in this case, a FootSLAM processor. All of them are able to generate a map of the areas they have visited, shown on their side. In this case, the central server is in charge of the generation and distribution of the combined map.

Figures 4 and 5 illustrate both of these alternatives for the online case. In the depicted cases, each pedestrian has direct access to her own individual map, but to be able to compute a combined map they need to communicate with the central server (centralized approach) or with the other pedestrians (decentralized approach) using the available network. The arrows indicate the possibility of communication between two pedestrians or a pedestrian and the central server. 


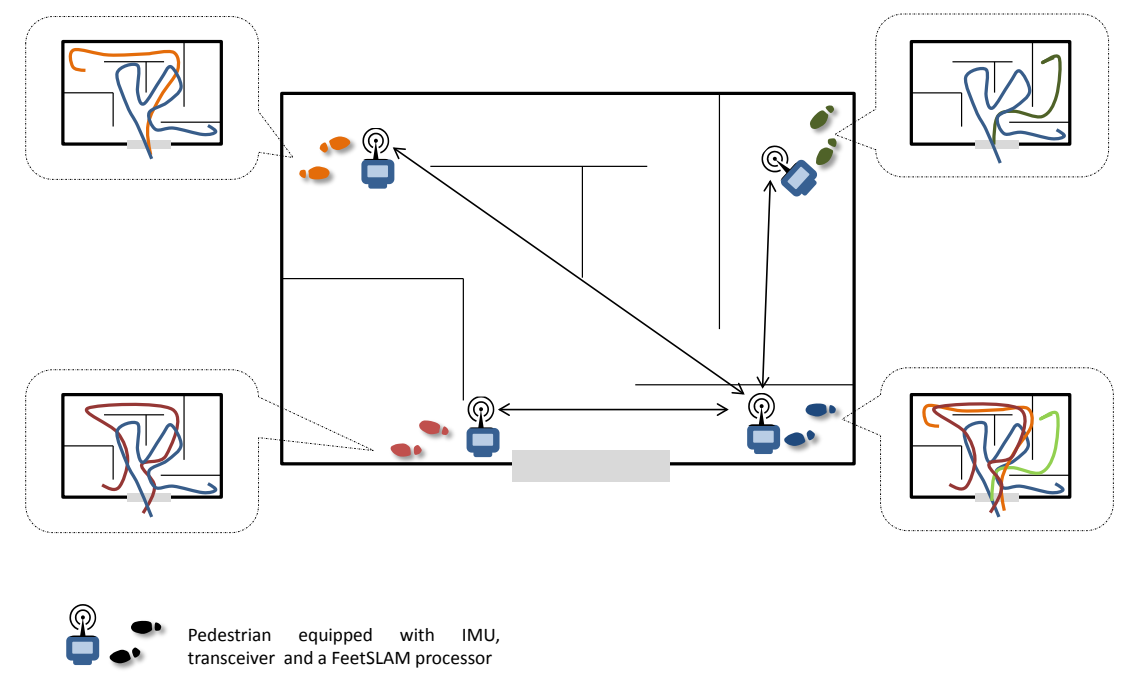

Fig. 5. Decentralized FeetSLAM: The four pedestrians have now access to the maps of some of the other pedestrians and are able to compute their own partial combined map. This map can be sent to the other pedestrians, helping all pedestrians have knowledge of the position of all the other pedestrians.

In any case, the pedestrians or the server in charge of the combination of the maps need to generate a table of contributing sources to avoid data sets using their own maps as priors. In that case, the map that arose from that specific data set can be subtracted from the combined map to obtain a fit prior map for the data set.

The main burden that FeetSLAM needs to face when computing a combined map is the need to transform the individual maps to place them within the same coordinate frame. The next section introduces some sensor fusion solutions to ease its time requirements.

\subsection{Sensor Fusion Solutions}

FootSLAM draws on the exclusive use of inertial sensors, but allows the integration of other sensors (sensor fusion) to help anchor the maps to a ground truth on the one hand, but also to increase their accuracy. This property can also be exploited by FeetSLAM to find the transformations that place all individual maps into the same coordinate system more quickly. The following approaches might be used:

- Use a GNSS receiver to anchor the map to a global coordinate system when possible (e.g. before entering the building). Due to GNSS inaccuracies, the individual maps still need to undergo transformation, but the area in which 
the transformation is searched can be drastically reduced, speeding up the combination process.

- Use the concept introduced by PlaceSLAM [14], whereby recognizable markers are used to help the FootSLAM algorithm converge. This could be markers already available in the environment, like WiFi or mobile network stations or optical features, or it could be markers which are manually placed in or around the building, e.g RFID tags or UWB stations, but whose position is known. Furthermore, if the position of another pedestrian or a potentially involved robot is known, this information can be used as a form of dynamic PlaceSLAM.

- Use the communication network through signal strength or time of arrival measurements between the pedestrians to get a rough distance estimation to support the needed transformation between their corresponding individual maps.

\subsection{Human-Robot Collaborative Mapping}

Hybrid solutions can also be used to face those cases in which the human intervention is partially prevented, such as a building in danger of collapsing, uncontrolled fire, hazardous materials, etc. In these scenarios, it can be useful to call robots into play. The robots can perform some parts of the exploration and undertake the mapping role. The following scenario is envisioned: The pedestrian performing the mapping task enters the building carrying a robot up until a point where the robot is set free. Then, the pedestrian exchanges his current location with the robot, who can start then performing his SLAM process, using visual sensors, PlaceSLAM or any available mapping and localization process. The robot can access and explore those areas where human life is threatened or where human access is not possible. Again, the whole process can be optionally anchored to a global coordinate system using a GPS receiver before entering the building. Figure 6 illustrates this idea.

\subsection{Influence on the Collaborating Pedestrians}

There exists a number of factors which have an influence on the mapping accuracy of FootSLAM, therefore affecting the resulting combined maps and the speed of the combination process:

- Number of users: the more users, the bigger the area that can be potentially mapped, but also the longer it takes to generate a combined map.

- Overlap of the areas visited by the pedestrians: overlap between the individual maps helps to find the best transformation between maps and also increases the convergence when using one as prior of the other.

- Duration of the walks (extension of the visited area and revisited area): the longer a walk lasts, the more the area is revisited (helping convergence) or the more area is explored (helping extensive mapping).

- Loop closure: closing loops helps the FootSLAM algorithm converge. 


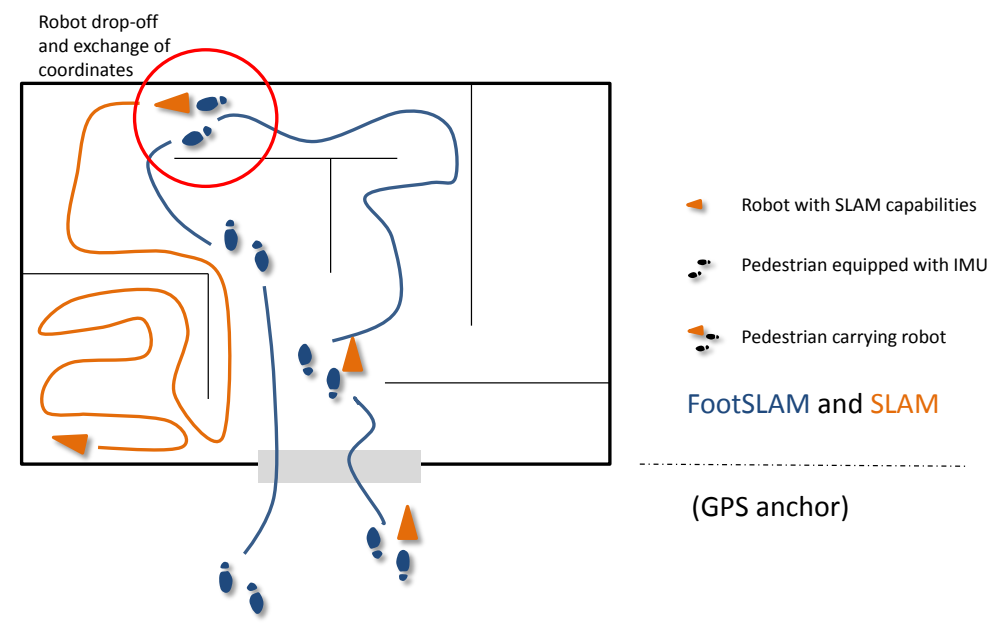

Fig. 6. Example of human-robot collaborative mapping: A pedestrian carrying a robot enters the building from the outside world optionally anchoring her position to the global coordinate system using a GPS receiver. Once inside the building, the pedestrian uses FootSLAM to generate a map of the areas she visits and locate herself within it. She reaches a certain point where she drops the robot off, which starts performing SLAM using the last coordinates of the pedestrian as starting point. The pedestrian leaves the building and the robot continues mapping the building autonomously.

- Use of other sensors: The more sensors are used in the mapping and navigation process, the more accurate the maps are.

Thus, the collaborating pedestrians could be instructed or rewarded to walk for a longer time in certain areas, to close loops, to revisit areas, to flag some markers, to use other sensors, etc. so that a more extensive and accurate combined map is generated.

\section{Conclusions and Further Work}

In this paper FootSLAM and its collaborative version FeetSLAM have been proposed to address the navigation challenges that arise in indoor environments in which the use of GNSS is prevented and a map is usually not available. FootSLAM and FeetSLAM allow the generation of a map of an indoor or underground area that can be used by rescue and emergency teams to localize themselves. Awareness of the relative position of the team members helps the coordination of the mission at hand, increasing the chances of its success. This has been illustrated with different cases of use for the online and for the offline scenarios. Two approaches have been presented to implement FeetSLAM: a centralized and a decentralized approach and the use of other sensors or robots has been explored to increment robustness, performance and safety. 
To experimentally validate the applicability of FeetSLAM to the different scenarios presented in this paper, further research needs to address online exchange of maps between the mapping agents or between the agents and a central server, that is, the definition of an ad-hoc network. Further work should also target the enhancement of the performance of the combined map generation and try to reduce its time demands. Likewise, the map generation abilities of FootSLAM in different kinds of environments or while the pedestrian runs, jumps, etc. is yet to be studied. Ongoing work is extending FootSLAM and FeetSLAM to three dimensions.

\section{References}

1. Foxlin, E.: Pedestrian tracking with shoe-mounted inertial sensors. In: IEEE Computer Graphics and Applications, vol. 25, no. 6, pp. 38-46 (2005)

2. Krach, B., Robertson, P., Angermann,M., Khider, M.: Inertial systems based joint mapping and positioning for pedestrian navigation. In: Proc. ION GNSS 2009, Savannah, Georgia, USA (2009)

3. Woodman, O., Harle, R.: Pedestrian localization for indoor environments. In: Proc. of the UbiComp 2008, Seoul, South Korea (2008)

4. Beauregard, S., Widyawan, Klepal, M.: Indoor PDR performance enhancement using minimal map information and particle filters. In: Proc. of the IEEE/ION PLANS 2008, Monterey, USA (2008)

5. Durrant-Whyte, H., Bailey, T.: Simultaneous Localization and Mapping: Part I Tutorial. In: IEEE Robotics \& Automation Magazine (2006)

6. Howard, A.: Multi-robot Simultaneous Localization and Mapping using Particle Filters. In: International Journal of Robotics Research, vol. 25, no. 12 (2006)

7. León, A., Barea R., Bergasa, L., López, E., Ocaña, M., Schleicher, D.: SLAM and Map Merging. In: Journal of Physical Agents, vol. 3, no. 1 (2009)

8. Lee, H., Lee, S., Lee. S., Lee, T., Kim, D., Park, K., Lee, K., Lee, B.: Comparison and Analysis of Scan Matching Techniques for Cooperative-SLAM. In: 8th International Conference on Ubiquitous Robots and Ambient Intelligence (URAI). Songdo Conventia, Incheon, Korea (2011)

9. Kleiner, A., Dornhege, C., Dali, S.: Mapping disaster areas jointly: RFIDCoordinated SLAM by Humans and Robots. In: Proceedings of the 2007 IEEE. International Workshop on Safety, Security and Rescue Robotics, Rome, Italy (2007)

10. Robertson, P., Garcia Puyol, M., Angermann, M.: Collaborative Pedestrian Mapping of Buildings Using Inertial Sensors and FootSLAM. In: ION GNSS 2011, Portland, Oregon, USA (2011)

11. Howe, J.: The Rise of Crowdsourcing. In: Wired, vol. 14, no. 6 (2006)

12. Brabham, D.: Crowdsourcing as a Model for Problem Solving: An Introduction and Cases. In: Convergence: The International Journal of Research into New Media Technologies, vol. 14, no. 1, pp. 75-90 (2008)

13. Dao, T., Zhou, Y., Thill, J., Delmelle, E.: Spatio-temporal location modeling in a 3D indoor environment: the case of AEDs as emergency medical devices. In: International Journal of Geographical Information Science, vol. 26, iss. 3 (2012)

14. Robertson, P., Angermann, M., Khider, M.: Improving Simultaneous Localization and Mapping for Pedestrian Navigation and Automatic Mapping of Buildings by using Online Human-Based Feature Labeling. In: Proc. IEEE/ION PLANS 2010, Palm Springs, CA, USA (2010) 